\title{
Nordiques
}

40 | 2021

Territoires de la migration dans les pays nordiques et baltes

\section{L'angoisse de la disparition : regard sur le paradoxe identitaire balte}

\section{Céline Bayou}

\section{(2) OpenEdition \\ Journals}

Édition électronique

URL : https://journals.openedition.org/nordiques/1279

DOI : $10.4000 /$ nordiques. 1279

ISSN : 2777-8479

Éditeur :

Association Norden, Bibliothèque de Caen la mer

Référence électronique

Céline Bayou, "L'angoisse de la disparition : regard sur le paradoxe identitaire balte », Nordiques [En ligne], 40 | 2021, mis en ligne le 02 mai 2021, consulté le 11 juillet 2021. URL : http://

journals.openedition.org/nordiques/1279; DOI : https://doi.org/10.4000/nordiques.1279

Ce document a été généré automatiquement le 11 juillet 2021.

Nordiques 


\title{
L'angoisse de la disparition : regard sur le paradoxe identitaire balte
}

\author{
Céline Bayou
}

\section{Introduction}

1 Depuis le début des années 1990, constat a été fait en Estonie, Lettonie et Lituanie d'un mouvement de décroissance démographique régulier. Les deux éléments à l'origine de cette tendance persistante sont l'accroissement naturel et les migrations. Mais ils n'interviennent pas dans les mêmes proportions : en effet, les taux de fécondité de ces pays sont comparables (voire meilleurs) que la moyenne européenne et, si l'espérance de vie y est en revanche moins bonne, cette contre-performance ne peut toutefois expliquer à elle seule l'ampleur de la décroissance.

2 Les statistiques migratoires (à manier avec précaution, celles concernant l'émigration étant par définition largement sous-estimées) révèlent en revanche, et ce depuis plus de 25 ans, un nombre d'émigrés traditionnellement supérieur à celui des immigrés (à l'exception de l'Estonie qui, récemment, semble vouloir inverser la courbe). Selon les autorités lituaniennes, l'émigration serait même responsable à $80 \%$ de la décroissance démographique. Or, parce que les populations qui quittent ces territoires sont en âge de procréer et de travailler, leur départ accentue le mouvement de vieillissement de la population, ce qui ne laisse pas d'inquiéter les gouvernements des trois pays, en premier lieu pour des raisons économiques. En effet, le manque de main-d'œuvre adaptée se fait sentir depuis longtemps, poussant les salaires à la hausse.

Dans des pays faiblement peuplés et où la population comme les dirigeants estiment que le risque d'une disparition pure et simple au cours du $\mathrm{XX}^{\mathrm{e}}$ siècle (effacement étatique par annexion et menace de disparition identitaire par immigration de populations étrangères) a été évité de justesse, cette situation n'est pas sans provoquer des crispations. Dès lors, plus qu'ailleurs sans doute, le débat sur l'accueil de migrants prend, dans ces trois pays, tournure d'interrogation introspective profonde: finalement, qu'est-ce qu'être Estonien, Letton ou Lituanien? Comment se définit cette 
identité intrinsèque et quelles solutions envisager pour, simplement, perdurer en tant que peuples?

\section{Le constat de la dépopulation}

Depuis le début des années 1990, les statistiques d'évolution de la population dans les trois pays révèlent une décroissance inexorable (voir tableau 1). Les projections confirment cette tendance, avec un léger apaisement qui amène toutefois, à horizon 2100, à une population de 1,145 million en Estonie, 1,081 million en Lettonie et 1,680 million en Lituanie. Si le taux de fécondité (nombre d'enfants par femme) en Europe est particulièrement faible (1,5 en 2020, contre 4,8 en Afrique subsaharienne), il connaît de forts écarts d'une région européenne à l'autre. La fécondité est en général plus élevée en Europe du Nord et plus faible en Europe du Sud ${ }^{1}$. Se rattachant au modèle nordique, les États baltes se situent légèrement au-dessus de la moyenne européenne (voir tableau 2) : leur fécondité est faible, mais moins alarmante que celle de certains pays du sud du continent. L'espérance de vie se situe en revanche dans la moyenne très basse de l'Union européenne (voir tableau 3), même si la part de la population âgée de plus de 65 ans est conforme, elle, à la moyenne européenne (autour de $20 \%)$.

Tableau 1. Évolution de la population et projections (en millions d'habitants)

\begin{tabular}{|l|c|c|c|c|c|c|c|c|c|}
\hline & $\mathbf{1 9 9 1}$ & $\mathbf{1 9 9 5}$ & $\mathbf{2 0 0 0}$ & $\mathbf{2 0 0 5}$ & $\mathbf{2 0 1 0}$ & $\mathbf{2 0 1 5}$ & $\mathbf{2 0 2 0}$ & $\mathbf{2 0 5 0}$ & $\mathbf{2 1 0 0}$ \\
\hline Estonie & 1,567 & 1,448 & 1,401 & 1,358 & 1,333 & 1,314 & 1,328 & 1,256 & 1,145 \\
\hline Lettonie & 2,658 & 2,500 & 2,381 & 2,249 & 2,120 & 1,986 & 1,907 & 1,395 & 1,081 \\
\hline Lituanie & 3,701 & 3,642 & 3,512 & 3,355 & 3,141 & 2,921 & 2,794 & 2,137 & 1,680 \\
\hline
\end{tabular}

Source : Eurostat

Tableau 2. Taux de fécondité (nombre d'enfants par femme)

\begin{tabular}{|l|l|l|l|l|l|l|l|}
\hline & $\mathbf{1 9 9 1}$ & $\mathbf{1 9 9 5}$ & $\mathbf{2 0 0 0}$ & $\mathbf{2 0 0 5}$ & $\mathbf{2 0 1 0}$ & $\mathbf{2 0 1 5}$ & $\mathbf{2 0 1 8}$ \\
\hline Estonie & 1,80 & 1,38 & 1,35 & 1,51 & 1,72 & 1,58 & 1,67 \\
\hline Lettonie & -- & -- & 1,24 & 1,38 & 1,35 & 1,70 & 1,60 \\
\hline Lituanie & 2,00 & 1,55 & 1,38 & 1,29 & 1,49 & 1,70 & 1,63 \\
\hline Moyenne UE-28 & -- & -- & -- & 1,51 & 1,61 & 1,58 & 1,55 \\
\hline
\end{tabular}

Source : Eurostat 
Tableau 3. Espérance de vie à la naissance (en années)

\begin{tabular}{|l|l|}
\hline & $\mathbf{2 0 1 8}$ \\
\hline Estonie & 78,5 \\
\hline Lettonie & 75,1 \\
\hline Lituanie & 76,0 \\
\hline Moyenne UE-28 & 81,0 \\
\hline
\end{tabular}

Source : Eurostat

5 En revanche, ce vieillissement de la population est largement accentué par les mouvements migratoires (voir tableaux 4 et 5, sachant que les statistiques d'émigration sont traditionnellement sous-estimées du fait de la non-déclaration de départ des sujets ${ }^{2}$ ). En effet, ceux qui partent sont dans leur grande majorité de jeunes adultes, en âge de procréer et de travailler ${ }^{3}$; leur émigration accentue donc le vieillissement de la population.

Tableau 4. Émigration

\begin{tabular}{|l|l|l|l|l|l|l|l|}
\hline & 1991 & 1995 & 2000 & 2005 & 2010 & 2015 & 2018 \\
\hline Estonie & -- & 9460 & 1784 & 4610 & 5294 & 13003 & 10476 \\
\hline Lettonie & 29729 & 16512 & 22911 & 17643 & 39651 & 20119 & 15814 \\
\hline Lituanie & 22503 & 25688 & 21816 & 57885 & 83157 & 44533 & 32206 \\
\hline
\end{tabular}

Source : Eurostat

Tableau 5. Immigration

\begin{tabular}{|l|l|l|l|l|l|l|l|}
\hline & 1991 & $\mathbf{1 9 9 5}$ & $\mathbf{2 0 0 0}$ & $\mathbf{2 0 0 5}$ & $\mathbf{2 0 1 0}$ & $\mathbf{2 0 1 5}$ & $\mathbf{2 0 1 8}$ \\
\hline Estonie & -- & -- & 35 & 1436 & 2810 & 15413 & 17547 \\
\hline Lettonie & 14684 & 2799 & 6483 & 6691 & 4011 & 9479 & 10909 \\
\hline Lituanie & 11828 & 2020 & 1510 & 6789 & 5213 & 22130 & 28914 \\
\hline
\end{tabular}

Source : Eurostat

Tableau 6. Solde migratoire

\begin{tabular}{|l|l|l|l|l|l|l|l|}
\hline & 1991 & 1995 & 2000 & 2005 & 2010 & 2015 & 2018 \\
\hline
\end{tabular}




\begin{tabular}{|l|l|l|l|l|l|l|l|}
\hline Estonie & -- & -- & -1749 & -3174 & -2484 & 2410 & 7071 \\
\hline Lettonie & -15045 & -13713 & -16428 & -10952 & -32640 & -10640 & -4905 \\
\hline Lituanie & -10675 & -23668 & -20306 & -51096 & -77944 & -42320 & -3292 \\
\hline
\end{tabular}

Source : calculs à partir des données Eurostat.

6 Aujourd'hui, on estime ${ }^{4}$ que, toutes diasporas confondues, $20 \%$ de la population lettone, $17 \%$ de la population lituanienne et $15 \%$ de la population estonienne ${ }^{5}$ vivent hors des frontières de leur pays. Mais, après des années de solde migratoire négatif depuis 1991 et un pic en 2010 avec une perte migratoire cumulée supérieure à 113000 (voir tableau 6), la tendance semble vouloir se stabiliser ou peut-être s'inverser (solde migratoire seulement légèrement déficitaire en 2018), l'Estonie ayant été la première à enregistrer un nombre plus élevé d'immigrants que d'émigrants au milieu des années 2010, avec une baisse du flux d'émigration et une hausse de celui d'immigration. Cette tendance demande à être confirmée, d'autant que la crise économique mondiale liée à la pandémie de Covid-19, en rebattant les cartes de l'évolution des marchés du travail, entrave pour le moment toute projection sérieuse. Depuis l'adhésion des trois pays à l'Union européenne ${ }^{6}$, la destination privilégiée à l'émigration des Estoniens reste la Finlande, pour d'évidentes raisons de proximité géographique et linguistique. Les Lettons et les Lituaniens, eux, se dirigent traditionnellement vers le Royaume-Uni (du moins jusqu'au Brexit), les États-Unis, l'Irlande, la Norvège et l'Allemagne ${ }^{7}$.

On constate une spécificité balte au regard de l'évolution démographique, qui accorde un poids particulièrement important au facteur migratoire. Cette donnée est appréhendée comme un risque par les autorités des trois pays, en particulier pour l'économie, puisqu'elle a des conséquences sur le marché du travail - notamment le taux de chômage et l'évolution des salaires -, sur la fiscalité, la sécurité sociale, le système des retraites - à horizon 2030 , on estime que près de la moitié de la population en Lettonie sera âgée de plus de 50 ans -, etc. Les difficultés les plus fréquemment évoquées sont liées d'une part à la pénurie de main-d'œuvre adaptée aux besoins - qui nécessiterait un recours à l'apport de main-d'œuvre étrangère - et d'autre part à la pression exercée sur les salaires, problème bien connu des États baltes pour avoir largement contribué à l'explosion de la violente crise économique de 2008.

\section{La crispation identitaire}

8 Mais ce tableau et les projections qui lui sont attachées sont aussi très souvent appréhendés dans les trois pays sous l'angle du risque identitaire. Si les autorités estoniennes, lettones et lituaniennes ne craignent pas sérieusement une disparition totale de ces nations, il arrive que le sujet soit évoqué, voire qu'il soit l'objet de pressions extérieures : c'est ainsi qu'en juin 2017 la chaîne télévisée russe NTV affirmait que « dans 100 ans, la nation lettone n'existera plus».

9 On peut quoi qu'il en soit parler à propos de ces trois pays d'angoisse existentielle, qui se traduit par une focalisation identitaire ${ }^{8}$ et la volonté permanente de consolider la nation. Les racines historiques de cette obsession sont parfaitement évidentes, liées à 
une existence en tant qu'États particulièrement courte pour deux d'entre eux (l'Estonie et la Lettonie ne sont apparues sur la scène internationale qu'à partir de 1918) et violemment niée au cours du XXe siècle puisque ces trois pays ont été rayés de la carte durant un demi-siècle du fait des occupations successives soviétique (1940-1941), nazie (1941-1944) puis soviétique (1944-1991). Au cours de cette période soviétique, le russe avait été érigé en langue officielle et l'afflux de populations immigrées en provenance du reste de l'URSS régulier et important. En Estonie par exemple, 1,6 million d'immigrés s'installèrent dans la république pendant les années soviétiques, faisant passer la part de la population russe de 8,2 \% en 1934 à 30,3 \% en $1989^{\circ}$. Pour l'Estonie, du fait de la russification culturelle qui l'accompagna, ce processus est considéré comme une colonisation.

10 Il en a résulté une crispation qui s'est traduite, en 1991, par les choix de l'Estonie et de la Lettonie de n'accorder automatiquement la citoyenneté qu'aux populations déjà estonienne et lettone avant les occupations et à leurs descendants. Les deux pays ont ainsi créé une nouvelle minorité (la majorité d'hier, russophone), population de noncitoyens vivant sur ces territoires et y payant leurs impôts, mais privés de certains droits à moins de passer un examen de citoyenneté. Cette ethnogenèse spécifique rend l'appréhension des populations de ces pays subtils, invitant à distinguer habitants, citoyens, non-citoyens et à démêler leurs identités ethnoculturelles.

11 À cette angoisse existentielle s'ajoute la volonté érigée en valeur de respecter la liberté de peuples qui en ont été longtemps privés. Objets, au cours de ces occupations, de mobilités forcées (déportations, exils) ou empêchées (interdiction de quitter l'URSS), les Baltes manifestent depuis 1991 et le rétablissement des indépendances une soif inextinguible de voir le monde, souvent pour se former ou pour y trouver un emploi mieux rémunéré. Alors que le déficit de main-d'œuvre s'est fait sentir très rapidement, les autorités unanimes des trois pays ont d'abord sciemment décidé d'adopter une politique peu - voire pas - interventionniste pour retenir ou faire revenir la population, au nom de cette valeur d'autant plus sacrée qu'elle avait été confisquée à ces peuples : la liberté.

12 L'autre versant de la question porte sur l'accueil de populations nouvelles qui pourraient abonder celle de ces pays. Et le paradoxe affleure, qui a pu être révélé notamment lors de la crise migratoire subie par l'Europe à partir de 2015 : bons élèves de l'Union européenne, les trois pays baltes se sont conformés au principe d'accueil d'un certain nombre de migrants. Mais cette perspective, plutôt qu'une solution au déficit de main-d'œuvre, a été largement perçue par les populations locales, alimentées en cela par les médias, comme un nouveau motif d'angoisse existentielle, rappelant là encore des souvenirs traumatiques liés au $\mathrm{XX}^{\mathrm{e}}$ siècle : la peur d'une disparition par dépassement numérique et dilution de l'identité.

\section{Quelles solutions au regard du défi migratoire?}

13 Quelles que soient les réticences, le constat de la dépopulation et du poids du facteur migratoire dans cette tendance de fond doit amener les autorités des trois pays à envisager des solutions. Trois chantiers s'ouvrent à elles : empêcher de partir, inciter à revenir et/ou accueillir de nouvelles populations. 


\section{Empêcher de partir}

laissé une empreinte qui ne semble pas devoir s'effacer avant longtemps, les autori laissé une empreinte qui ne semble pas devoir s'effacer avant longtemps, les autorités ont donc d'abord refusé d'intervenir, lorsqu'elles ont constaté, dès le début des années 1990, qu'une part importante de la population quittait le pays à l'indépendance recouvrée pour trouver ailleurs une vie matérielle plus attrayante. Interrogée en tant que chef de l'État sur les mesures à prendre pour stopper cette hémorragie préjudiciable à l'économie, la Présidente lettone Vaira Viķe-Freiberga (1999-2007) s'est à plusieurs reprises expliquée sur le non-interventionnisme pour lequel elle avait opté durant ses mandats : il lui semblait inenvisageable de prendre des mesures pour retenir ceux qui aspiraient au départ et n'avaient pas l'héroïsme de supporter par exemple des chutes brutales de salaire (de 20 à $40 \%$ en 2008 lors de la crise économique) ou l'absence de perspective du marché du travail, précisément parce que le peuple letton, cinquante ans durant, avait été privé de ce droit d'aller et venir. Alors, quitte à affaiblir cet État qui avait pourtant tant besoin de forces vitales, elle préférait placer la liberté au-dessus de toute conjecture, y compris peut-être au-dessus du patriotisme. De la même façon, V.Viķe-Freiberga a défendu cette liberté de circulation devant ses partenaires européens au moment de l'ouverture des frontières, quitte à en bousculer certains: «Ce n'est ni le plombier polonais ni le médecin letton qui présentent des menaces, mais le monde qui tourne!», lâchait-elle en 2004 afin de dénoncer les réticences de certains pays de la vieille Europe à laisser entrer sur leurs territoires ces migrants d'un genre nouveau ${ }^{10}$. Cette obsession du respect de la libre circulation s'est également exprimée en 2020, lorsque la pandémie de Covid-19 a amené à la fermeture de nombre de frontières, y compris en Europe : les États baltes, acculés eux aussi, s'y sont pliés, mais de mauvaise grâce, en dénonçant haut et fort l'impuissance d'une UE qui, selon eux, manquait symboliquement à l'un de ses engagements prioritaires ${ }^{11}$.

Pourtant, au vu de l'ampleur de la dépopulation, les discours ont peu à peu évolué et les autorités ont réfléchi aux moyens qui permettraient de donner envie aux populations de rester dans leur pays. Elles invoquent ainsi l'enjeu de retenir par l'attractivité et développent depuis quelques années un discours sur le fait que chaque individu a de la valeur: concrètement, se mettent peu à peu en place des plans de formation professionnelle afin de mieux adapter la main-d'œuvre aux besoins de l'économie; de même, la création d'entreprises est facilitée dans les trois pays. Mais ces outils, économiques, se révèlent peu efficaces, confrontés à l'impossible équation entre attraction des salaires et enjeu de la productivité du travail. Alors certains ont envisagé une solution purement statistique quitte à transcender parfois la pourtant si délicate question ethnique: l'ancien président letton Raimonds Vējonis (juillet 2015juillet 2019) a ainsi beaucoup œuvré pour que la citoyenneté lettone soit automatiquement attribuée dès leur naissance aux enfants nés de non-citoyens (il s'agit ainsi de cesser de créer des non-citoyens, ceux-ci représentant environ $11 \%$ de la population en Lettonie et $6 \%$ en Estonie). La Lituanie, elle, a longtemps débattu de l'octroi de la double citoyenneté à l'intention de ceux qui sont partis et, tout particulièrement, des enfants d'émigrés nés ailleurs que sur le sol lituanien (mais ethniquement lituaniens). Au risque du paradoxe, il s'agit bien de comptabiliser de plus en plus de citoyens, quelles que soient leur ethnicité ou leur localisation. 


\section{Faire revenir?}

16 des bienfaits d'un retour. En 2010-2012, l'Estonie a par exemple lancé un programme baptisé "Faire rentrer les talents à la maison», destiné aux plus qualifiés. Non seulement il a partiellement échoué auprès de sa cible, mais il a contribué à éloigner de l'objectif les moins qualifiés, blessés de se sentir exclus ${ }^{12}$. Il est clair que les incitations au retour sont aujourd'hui bien moins importantes qu'au début des années 1990 : le rétablissement des indépendances a constitué un puissant facteur d'attraction pour des diasporas qui avaient émigré sous la contrainte. Aujourd'hui, alors que l'aiguillon de l'émigration, qu'il soit éducatif et économique, est largement basé sur le volontariat, la charge symbolique d'un potentiel retour perd en intensité.

17

Estonie, en Lettonie et en Lituanie à l'égard de la diaspora ont jusqu'à aujourd'hui plus porté sur le maintien de la langue et de la culture que sur de véritables argumentaires incitant au retour. Le Programme des compatriotes mis en place en 2004 en Estonie a ainsi pour priorités l'enseignement de la langue estonienne à l'étranger, la préservation de la culture et de la connaissance de l'histoire, le sens de l'appartenance à la nation estonienne... et le retour des expatriés ; mais le fait qu'il soit géré conjointement par les ministères de l'Éducation et de la Culture révèle ses véritables objectifs qui restent le maintien du lien identitaire avec la diaspora. La Lituanie est plus active en la matière, avec notamment le site internet "Je choisis la Lituanie", initialement dédié à l'information sur les conditions du retour. On note toutefois qu'au cours des dernières années, il s'est élargi dans l'information adressée à tout candidat à l'immigration, qu'il soit Lituanien ou pas. Quant à la Lettonie, dotée depuis janvier 2019 d'une nouvelle loi sur la diaspora qui s'apparente dans son contenu à celle décrite dans le cadre du programme estonien, mais est gérée par le ministère des Affaires étrangères, elle tend à élargir le concept de "diaspora ", outre aux citoyens lettons vivant à l'étranger, aux citoyens ayant une " connexion durable » avec la Lettonie ainsi qu'aux membres de leur famille. Les membres de la diaspora lettone ont été, en revanche, autorisés dès 2013 à adopter une double citoyenneté s'ils vivent dans des pays de l'UE et de l'OTAN (ainsi qu'en Australie, Nouvelle-Zélande et au Brésil).

Le frémissement perçu dans les chiffres de l'immigration depuis 2015 en Estonie s'explique partiellement par ce mouvement de retours, motivés par une hausse régulière des salaires, une croissance économique notable, mais également les difficultés rencontrées sur le marché du travail dans le pays d'émigration, le cas du Royaume-Uni apparaissant évidemment comme spécifique en la matière: la perspective du Brexit et les incertitudes qui y étaient attachées au regard des droits de la main-d'œuvre immigrée ont certainement incité nombre de Baltes à organiser leur retour dans la mère-patrie.

Il n'en reste pas moins que la politique de ces pays à l'égard de la diaspora s'apparente plus à un effort pour garder le lien qu'au déploiement d'une authentique stratégie pour l'inciter à rentrer. Il est probable en outre que la vague d'émigration liée aux premières années de l'indépendance puis à l'adhésion à l'UE et à l'intégration dans l'espace Schengen a revitalisé la diaspora plus ancienne, contribuant à son assise en tant que telle ${ }^{13}$. Si, au cours des années 1950 ou 1970, les diasporas baltes à l'étranger se réunissaient autour de l'espoir un peu fou d'un retour lié à l'hypothétique 
effondrement de l'URSS, aujourd'hui les associations de diasporas s'animent autour de l'enjeu de préserver la culture et la langue de naissance sans envisager une réinstallation sur le territoire originel. Tout comme à la fin $\mathrm{du} \mathrm{XIX}^{\mathrm{e}}$ et au début du $\mathrm{XX}^{\mathrm{e}}$ siècles, avant la naissance des États estonien et letton, on pouvait se sentir Estonien à Riga ou Letton à Saint-Pétersbourg, on peut donc aujourd'hui être Estonien et consolider son identité estonienne sans envisager l'«estonité » par rapport au territoire. C'est le cas, par exemple, du rassemblement annuel d'Estoniens de la province d'Ontario, réuni au sein de l'association Kotkajärve Metsaülikool (université sylvestre du lac de l'aigle ${ }^{14}$ : à l'été 2017 , pour fêter son $50^{\text {ème }}$ anniversaire, l'association a adopté une déclaration sous forme d'appel afin que ses membres soient reconnus comme peuple indigène de ce territoire. Créée durant la période d'occupation soviétique, l'association a toujours eu pour mission de préserver la culture et la langue estoniennes. Estimant que le territoire d'accueil est devenu leur base ethnique et linguistique et partant du constat que les peuples indigènes sont les plus efficaces à protéger l'environnement, l'héritage culturel et les langues, et ce à travers le monde, ses membres ont également demandé la création de districts électoraux pour les citoyens expatriés, id est pour ces Estoniens émigrés qui n'ont pas abandonné leur héritage, mais estiment qu'ils doivent désormais être perçus comme une ressource potentielle permettant de renforcer la présence et l'influence de l'Estonie dans le monde. On assiste donc à une véritable offre de déterritorialisation.

\section{Accueillir ?}

Pour combler le vide, une autre solution peut être le recours à l'immigration de populations étrangères. Mais le sujet est sensible dans ces trois pays qui relèvent d'une région historiquement multiethnique, où les peuples ont dû effectuer leur ethnogenèse (voire, pour certains, construire leur État) au contact, parfois hostile, d'autres peuples (six siècles de présence germanique notamment, mais aussi suédoise, polonaise, russe...) Cette histoire a certes été productrice d'identité : Yves Plasseraud évoque un " creuset baltique ${ }^{15}$, comprenant aussi des Russes, des Germano-Baltes, des Polonais, des Juifs..., qui a généré des frottements, souvent pacifiques mais parfois aussi dans le sang, entre ces peuples et qui leur ont permis de forger leur identité propre (et, accessoirement, de construire leur image de l'Autre). En sont nées des identités spécifiques qui ont intériorisé ces difficultés : après 1991, lorsque ces États ont eu à choisir leur modèle de reconstruction, cette mémoire de la multiethnicité a joué son rôle. Aujourd'hui, ces nations ne sont pas "civiques", sur le modèle français (où se maintient la confusion entre citoyenneté et nationalité), mais, comme la plupart de celles d'Europe centrale, elles sont «ethniques»: l'État se veut l'État de la nation éponyme et ceux qui n'appartiennent pas à la nation titulaire sont des "minoritaires ", qualité imposée par leur ethnicité. En Estonie et en Lettonie, certains ne possèdent pas même la citoyenneté, ce qui confère à ces immigrés d'hier une qualité d'existence qui fait écho à ce que Iain Chambers qualifie d'identités "dénuées" (d'accès à la citoyenneté, à la justice, à certains emplois, etc. $)^{16}$

Il convient de souligner que, par sa violence, la période 1945-1991 a été particulièrement traumatique pour ces régions, soumises à une immigration russophone (populations russes, biélorusses, ukrainiennes notamment) régulière et importante qui a totalement bouleversé la structure ethnique (en 1989, les Lettons ethniques ne représentaient plus que $52 \%$ de la population de leur République) et a fait 
naître l'angoisse de la disparition en tant que nation (après celle de l'État). En 1991, l'alternative consistait à faire le choix de la reconstruction des États ethniques d'avantguerre ou de reconnaitre officiellement le multiculturalisme existant. Certes, le processus a évolué en près de trente ans, mais les citoyens de cultures différenciées sont, dans ces trois pays, résolument invités à se fondre dans l'anonymat de sociétés de plus en plus civiles ${ }^{17}$.

22 L'angoisse existentielle qui perdure rend ces peuples peu enclins à promouvoir l'immigration. En 2015, lorsque l'Europe s'est trouvée totalement dépassée par un afflux de migrants qu'elle n'avait pas anticipé, les débats ont été houleux au sein de la classe politique des trois pays. L'ex-Présidente lettone V. Viķe-Freiberga n'a pas faibli sur ses principes et a rapidement mis en garde contre le «cirque politique » que cette situation risquait d'engendrer dans son pays : "Si nous affirmons que nous sommes incapables d'accueillir correctement un si petit nombre de réfugiés et de les intégrer dans notre société, alors cela reviendra, pour la Lettonie, à signer son propre certificat de pauvreté et à se discréditer toute seule. ${ }^{18}$ Mais son inquiétude ne s'arrêtait pas là : depuis que la Russie prétend se préoccuper du sort des russophones qui vivent hors de ses frontières, quitte à envisager de venir les défendre où qu'ils soient, la Lettonie n'a vraiment pas intérêt à exprimer des doutes quant à sa capacité à intégrer des populations non lettones en son sein. À moins de donner carte blanche à Vladimir Poutine pour s'ingérer dans les affaires du pays. L'accueil des migrants syriens, afghans et africains relevait donc, pour elle, de la sécurité du pays.

Les populations des trois pays ont exprimé de fortes réticences au sujet de l'accueil des réfugiés. Lorsque l'UE a imposé un système de répartition par quotas, il a été accepté par les gouvernements au nom de la solidarité européenne, mais en faisant état de leurs doutes concernant leur manque d'infrastructures d'accueil et d'expérience... Jamais cet accueil n'a été appréhendé sous l'angle de l'opportunité qu'aurait pu constituer cet apport de population. L'ampleur des débats liés dans ces pays à l'installation de quelques centaines de réfugiés qui, pour la plupart, ne sont d'ailleurs pas restés longtemps sur ces territoires s'est apparentée selon certains à une véritable " tempête dans un verre d'eau ${ }^{19}$ : les arguments des opposants (partage avec d'autres d'acquis sociaux fragiles, impossible intégration de populations musulmanes, islamisation des sociétés européennes, débat sur le port du voile, manque d'éducation des demandeurs d'asile ou non-respect des droits humains $\left.{ }^{20} . ..\right)$ ont d'abord mis au jour une peur de l'inconnu qu'on a retrouvée dans bien d'autres pays; à ceci près qu'elle n'aurait finalement pas eu prise longtemps dans les pays baltes ${ }^{21}$ et aurait vite dévié sur la mention de la "colonisation » subie durant la période soviétique. Ces débats ont fait le lit, avec plus ou moins de bonheur, des partis les plus extrêmes; ils ne sont sans doute pas étrangers à l'arrivée au pouvoir en Estonie du parti d'extrême droite EKRE. Celui-ci se présente en défenseur de la nation estonienne et de son identité et, sans surprise, défend les thèses les plus restrictives concernant l'accueil d'immigrés : critique virulent du système communautaire de répartition par quotas - assimilé par lui à une politique d'immigration de masse et d'islamisation sous la bannière de l'ouverture -, EKRE a bénéficié lors des élections législatives de mars 2019 de 17,8 \% des voix, lui permettant d'entrer dans un gouvernement de coalition. Pour le parti, la seule politique migratoire qui vaille en Estonie serait celle qui permettrait d'augmenter le montant et le pourcentage d'Estoniens en Estonie afin d'éviter une dilution de l'identité, sur le modèle prôné par Bruxelles ${ }^{22}$. Il s'agit en particulier de persuader les Estoniens partis travailler en Finlande voisine de rentrer, l'enthousiasme d'EKRE étant moins évident 
lorsqu'il s'agit de pays européens plus éloignés, qui auraient déjà pervertis par leur idéologie l'esprit des immigrés. Le parti soutient également l'arrêt du recours à une main-d'œuvre saisonnière, celle-ci venant principalement d'Ukraine et d'autres pays de l'espace post-soviétique.

Tallinn a mis en place en 1993 un système de quota annuel, équivalent à $0,1 \%$ de la population du pays, autorisé chaque année à venir travailler en Estonie et pouvant bénéficier d'un permis de résidence (ce quota n'inclut pas, notamment, les travailleurs saisonniers et les ressortissants des pays de l'UE). Ainsi, 1314 personnes ont obtenu un permis de résidence en 2020, au terme d'un examen précis de leur niveau d'études et des secteurs jugés prioritaires parce que souffrant de pénurie de main-d'œuvre (à savoir santé, éducation, information et communication, transports et, enfin, industrie). Le problème est que, chaque année, le quota est atteint de plus en plus tôt. Pour autant, il n'augmente pas (pour 2021, il a été fixé à 1315 personnes), comme si son élargissement allait provoquer un débat dans le pays ou mettre ce dernier en danger. De son côté, la Lituanie a mis en place en janvier 2019 une task force pour l'intégration d'abord dédiée à l'immigration de retour et vite passée à la promotion de l'immigration de populations diplômées. La Lettonie semble la moins active des trois pays sur la question de l'immigration, qui n'apparait pas comme une priorité donc; pour le moment, les politiques publiques restent centrées sur la gestion de la diaspora et son éventuel retour.

On se trouve donc en présence d'un paradoxe commun aux trois pays: l'ampleur de l'émigration en provenance de ces États est telle qu'elle est perçue comme mettant en danger la consolidation de leurs identités respectives. Ce risque pourrait être aisément prévenu par une politique de promotion de l'immigration, mais cette solution est peu prisée - les non-dits à son sujet sont parlants, quand elle n'est pas ouvertement rejetée - parce qu'appréhendée avant tout comme un facteur de risque culturel - et tout particulièrement linguistique - pour l'identité. Le fait que la majorité des candidats à l'immigration, notamment saisonnière, dans ces trois pays soient des ressortissants de l'espace post-soviétique (et donc des russophones) est un facteur de rejet particulièrement bien intégré au sein de la population: l'accueil de ces populations n'est pas l'objet d'un débat public houleux, mais les trois pays se sont dotés de gouvernements suffisamment nationalistes pour que les électeurs soient confiants dans le fait que l'option d'un recours massif à une main-d'œuvre russophone soit unanimement perçue comme une menace et non comme une solution. La peur du dépassement numérique reste solidement ancrée. Spécificité de la construction identitaire des trois pays, la langue est en effet depuis des siècles la ressource identitaire privilégiée (l'éveil national a pu se faire par appropriation de la langue). Dans cette équation, on ne peut faire entrer l'immigration de retour des diasporas qui apparaît quasiment comme un "non-sujet » : parce que la question de l'identité est liée à la langue, il y a consensus au sein de ces pays pour considérer que la priorité n'est pas tant d'y soutenir l'économie que d'en promouvoir la langue, où que ce soit.

\section{Conclusion : l'essentialisation de la langue, la disparition du territoire}

La mémoire collective des trois pays, obsessionnelle, fait donc planer le spectre d'une nouvelle domination linguistique s'il y a politique d'immigration, perçue comme un 
risque majeur pour le maintien des identités locales. Cette essentialisation de la langue peut transcender les frontières, ce qu'on a constaté sur la question de l'immigration de retour (plutôt qu'à faire rentrer les diasporas, les autorités sont attelées à la tâche de maintenir l'identité culturelle, donc linguistique, de ses représentants et de leurs descendants). Pourtant, l'appartenance communautaire qui s'exprime fréquemment à travers la spatialité présente en général l'avantage de pouvoir s'ancrer dans un réel matérialisé via la localisation, les paysages, la présence de limites, de hauts lieux, etc. Le processus d'identification peut s'en trouver facilité. Dès lors, le territoire est appréhendé à la fois comme support identitaire et comme producteur d'identité.

À cet égard, l'Estonie se présente comme une sorte de cas extrême: les ambitions numériques de ce pays, désormais bien connues, dépassent indéniablement la seule ambition qu'on pouvait leur prêter encore il y a quelques années en matière de branding, pour traduire aussi un souhait de déterritorialisation de l'identité. On peut citer l'expérience inédite, lancée en 2014, d'une e-résidence qui, en avril 2021, avait déjà séduit plus de 79000 personnes dans plus de 170 pays, leur permettant de créer et gérer plus de 12000 entreprises « estoniennes " sans avoir, à quelque moment que ce soit, à se trouver physiquement en Estonie. De même, en 2018, les autorités estoniennes ont annoncé l'ouverture d'une e-ambassade destinée à accueillir des serveurs sécurisés hors des frontières de l'Estonie - en l'occurrence sur le territoire du Luxembourg - afin de protéger les données des sites du Parlement estonien, de ministères, de banques ou de médias. L'association citée plus haut et sise dans l'Ontario a, elle, proposé de créer un web estonien, baptisé Estica, qui serait développé conjointement par des autochtones et des expatriés. Enfin, le fondateur du projet d'e-résidence, Kaspar Korjus, devenu en 2019 Directeur général du projet «Borderless Nation States », a un moment évoqué la possible création d'une crypto-monnaie estonienne, le EstCoin... Justifiées par des intérêts économiques (notamment les projets d'e-résidence et de crypto-monnaie) ou sécuritaires (à cet égard l'e-Ambassade révèle l'intériorisation par les autorités estoniennes de la vulnérabilité du territoire estonien, justifiant la mise à l'abri hors des frontières des données essentielles de l'État), ces initiatives affichent le rapport distancé entretenu par ce pays à son territoire national, contredisant l'idée selon laquelle l'identité est toujours située : «Ce que nous sommes ne peut être séparé 'd'où nous sommes'", affirmait ainsi Alain Dieckhoff ${ }^{23}$. Le «où nous sommes" des promoteurs de l'e-Estonie semble ne s'imposer aucune limite, quitte à oblitérer le patrimoine identitaire géographique attaché à chacun ${ }^{24}$.

Cette approche jusqu'au-boutiste qui paraît vouloir fusionner identité nationale (via la langue) et identité étatique (que la numérisation permet de déterritorialiser) peut évidemment être intégrée dans le processus de mondialisation, qui elle-même bouscule les identités et soulève la question de leur devenir. Le brassage accéléré qui enveloppe désormais tout un chacun appelle bien une nouvelle conception de l'identité. Il y a plus de vingt ans, Amin Maalouf notait déjà avec une certaine inquiétude que nous ne pourrions nous contenter d'imposer aux milliards d'êtres humains le choix entre affirmation outrancière de leur identité et perte de toute identité, id est «entre l'intégrisme et la désintégration "; dès lors, estimait-il, nous devrions impérativement être encouragés à assumer nos identités multiples, à moins de prendre le risque de former des légions de fous sanguinaires et d'égarés ${ }^{25}$.

L'option numérique de l'Estonie, résolument située de plain-pied dans la mondialisation, bouleverse totalement l'expression identitaire classique. Elle révèle 
une autre ambition encore : pour K. Korjus, il s'agit bel et bien de créer une «nation numérique ", en donnant par exemple aux e-résidents le sentiment de faire partie d'une communauté. Si les concepteurs du projet parlent bien estonien, ses adeptes n'utilisent que l'anglais et l'identité promue se passe à la fois de territoire et de langue : cette identité est virtuelle et mondiale. Surtout, elle confère à l'Estonie une identité qu'on pourrait considérer comme aboutissement ultime de consolidation: celle de créatrice d'identité par le lancement d'une nouvelle nation, numérique.

Si la nécessité, soulignée par A. Maalouf, d'intégrer des identités multiples se vérifie aisément par le fait que, depuis des siècles, l'être humain s'accommode aisément d'appartenances multiples, la question peut toutefois être posée de savoir à laquelle va sa principale loyautée ${ }^{26}$. Les États qui en sont conscients défendent certains marqueurs ayant fait la preuve, au cours de l'histoire, de leur primauté: si être l'Estonie aujourd'hui, c'est partiellement effacer la primauté du territoire, c'est parce que l'expérience historique a prouvé que la nation pouvait y survivre. Le marqueur linguistique, en revanche, demeure prioritaire pour les autorités. Au regard des phénomènes migratoires, être estonien demeure lié, a minima, au fait de maîtriser la langue estonienne, dans les frontières du pays ou au-delà.

\section{NOTES}

1. Gilles Pison, «France : la fécondité la plus élevée d'Europe », Population et sociétés, n 575 , 3/2020, p. 1-4.

2. Gilles Pison rappelle que peu de pays disposent de système permettant d'observer les départs de migrants de leur territoire; les statistiques migratoires sont donc produites à partir de l'observation des arrivées dans les pays d'accueil. Gilles Pison, «Le nombre et la part des immigrés dans la population: comparaisons internationales", Population et sociétés, $n^{\circ} 563$, 2019/2, p. 8.

3. Godfried Engbersen, Joost Jansen, «Emigration from the Baltic States : Economic impact and policy implications ", in Coping with Emigration in Baltic and East European Countries, OECD, 2013, p. 15-16.

4. Ieva Birka, «Can Return Migration Revitalize the Baltics? Estonia, Latvia, and Lithuania Engage Their Diasporas, with Mixed Results », Washington, DC, Migration Policy Institute, 8 mai 2019.

5. On parle ici de populations estonienne, lettone et lituanienne définies au regard de leur lieu de naissance.

6. Au début des années 1990, les trois pays ont connu un départ massif de populations russophones, qui ont quitté les nouveaux États estonien, letton et lituanien pour s'installer en Russie, Ukraine, Biélorussie... en fonction de leur histoire personnelle et familiale.

7. Mihails Hazans, "Emigration From Latvia : A Brief History and Driving Forces in the TwentyFirst Century", in Rita Kaša, Inta MieriN̦a (eds) The Emigrant Communities of Latvia, IMISCOE Research Series. Springer, 2019, p. 35-68. 
8. Victoria Krebber, Identity Questions in the Baltic States - An Analysis of Estonia, Latvia and Lithuania regarding their Attitudes towards a European Identity, Bachelor Thesis, University of Twente, septembre 2012, $70 \mathrm{p}$.

9. Sur la même période, 1,26 million de personnes quittèrent la république estonienne, ce qui provoqua au final un apport migratoire de 340000 personnes. Jaak Valge et Anu Raudsepp, « La migration vers l'Estonie après la Seconde Guerre mondiale », in Uta Fenske, Daniel Groth, KlausMichael Guse, Bärbel P. Kuhn (dir.), Colonisation et décolonisation dans les cultures historiques et les politiques de mémoire nationales en Europe, Bruxelles, Peter Lang, 2015, p. 173.

10. Interview accordée à l'auteure. Cité in Céline Bayou \& Éric Le Bourhis, Les Lettons, Paris, Ateliers Henry Dougier, 2016, p. 27.

11. Céline Bayou, «Les États baltes à l'épreuve du Covid-19: une certaine idée de l'Europe ", The Conversation, 19 mai 2020.

12. Ieva Birka, op. cit. note 4.

13. Emily Gilbert, Rebuilding post-war Britain - Latvian, Lithuanian and Estonian refugees in Britain, 1946-51, Malte, Pen \& Sword Books Ltd, 2017, p. 204-217.

14. «Kotkajärve Metsaülikool calls for Estonians to be recognised as indigenous people ", Estonian World, 5 septembre 2017.

15. Yves Plasseraud, "Ethnogenèse et création d'États : le cas de l'aire baltique ", Sens public, 2 février 2007.

16. Iain Chambers, Migrancy, Culture, Identity, Londres, Routledge, 1994, $172 \mathrm{p}$.

17. Yves Plasseraud, Les États baltiques - Les sociétés gigognes, Crozon, Ed. Armeline, 2003, p. 355-357.

18. Céline Bayou et Éric Le Bourhis, op. cit., p. 28-29.

19. Aldis Austers, "Immigration and refugees in the European Union and Latvia ", in Latvian Foreign and Security Policy Yearbook 2017, Latvian Institute of International Affairs, Riga, 2017, p. 116.

20. Maria Golubeva et Marta Rikša, « Asylum seekers in Latvia », Riga, Providus, novembre 2015.

21. Annual Review, Estonian Internal Security Service, Tallinn, 2016, p. 5.

22. Voir l'interview de Vello Pettai par Arch Puddington, «In Liberal Estonia, Rigth-Wing Populists Are Making Their Mark», Freedom House, 2 juillet 2019 et Viljar Veebel, "The Rise of Rigth-Wing Populists in Estonia », Foreign Policy Research Institute, 31 juillet 2019.

23. Alain Dieckhoff, La Nation dans tous ses États - Les identités nationales en mouvement, Paris, Flammarion, 2000, p. 44.

24. France Guérin-Pace, "Sentiment d'appartenance et territoires identitaires ", L'espace géographique, tome 35, n 4, 2006, p. 299 et F. Guérin-Pace, «Lieux habités, lieux investis : le lien au territoire, une composante identitaire?", Économie et statistique, n 393-394, 2006, p. 2.

25. Amin Maalouf, Les identités meurtrières, Paris, Grasset, 1998, p. 49.

26. Tzvetan Todorov, La peur des barbares - Au-delà du choc des civilisations, Paris, Robert Laffont, 2008, p. 118.

\section{RÉSUMÉS}

Les pays baltes (Estonie, Lettonie, Lituanie) sont confrontés à un phénomène persistant de dépopulation qui les conduit à s'inquiéter pour la pérennité de leur existence en tant que 
nations. Les migrations (émigration continue et immigration faible) étant les facteurs principaux à l'origine de cette perte de population, les trois pays semblent placés devant trois solutions possibles : empêcher de partir, inciter à revenir et/ou accueillir de nouvelles populations. C'est sans compter sur le paradoxe balte qui définit l'identité avant tout par la langue et laisse une place secondaire au territoire.

The Baltic countries (Estonia, Latvia, Lithuania) are faced with a persistent phenomenon of depopulation which leads them to worry about the sustainability of their existence as nations. As migrations (continuous emigration and low immigration) are the main factors behind this loss of population, the three countries seem to be faced with three potential solutions: to prevent leaving, to encourage return and/or to welcome new populations. This is without taking into account the Baltic paradox which defines identity above all through language and leaves a secondary place to territory.

INDEX

Keywords : emigration, immigration, depopulation, demography, Baltic states

Mots-clés : émigration, immigration, dépeuplement, démographie, pays baltes

\section{AUTEUR}

\section{CÉLINE BAYOU}

Céline Bayou est chargée de cours à l'Institut national des langues et civilisations orientales (INALCO), chercheure associée au Centre de recherches Europes-Eurasie (CREE - INALCO) et rédactrice en chef de Regard sur l'Est (www.regard-est.com). 